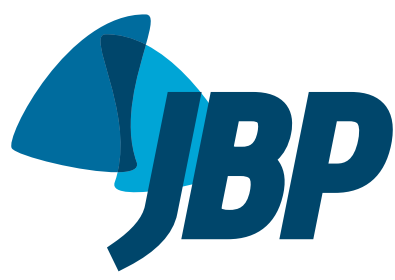

1. Programa de Pós-Graduação em Ciências Pneumológicas, Universidade Federal do Rio Grande do Sul - UFRGS - Porto Alegre (RS) Brasil.

2. Laboratório de Vias Aéreas e Pulmão, Hospital de Clínicas de Porto Alegre HCPA - Porto Alegre (RS) Brasil.

3. Hospital de Clínicas de Porto Alegre HCPA - Porto Alegre (RS) Brasil.

a. (D) http://orcid.org/0000-0001-8992-2607

b. (iD) http://orcid.org/0000-0001-6820-2387

c. (D) http://orcid.org/0000-0003-3001-1731

d. (iD) http://orcid.org/0000-0002-1379-6912

e. (iD http://orcid.org/0000-0002-6431-3079

Submitted: 7 February 2018

Accepted: 12 August 2018.

Study carried out under the auspices of the Programa de Pós-Graduação em Ciências Pneumológicas, Universidade Federal do Rio Grande do Sul - UFRGS -

Porto Alegre (RS) Brasil.

\section{Effect of vaporized perfluorocarbon on oxidative stress during the cold ischemia phase of lung graft preservation}

\author{
Renata Salatti Ferrari1,a, Leonardo Dalla Giacomassa Rocha Thomaz ${ }^{2, b}$, \\ Lucas Elias Lise Simoneti ${ }^{2, c}$, Jane Maria Ulbrich ${ }^{1,3, d}$, Cristiano Feijó Andrade ${ }^{1,3, e}$
}

\begin{abstract}
Liquid perfluorocarbon (PFC) instillation has been studied experimentally as an adjuvant therapy in the preservation of lung grafts during cold ischemia. The objective of this study was to evaluate whether vaporized PFC is also protective of lung grafts at different cold ischemia times. We performed histological analysis of and measured oxidative stress in the lungs of animals that received only preservation solution with low-potassium dextran (LPD) or vaporized PFC together with LPD. We conclude that vaporized PFC reduces the production of free radicals and the number of pulmonary structural changes resulting from cold ischemia.
\end{abstract}

Keywords: Ischemia; Reperfusion; Fluorocarbons; Lung transplantation; Oxidative stress.
Various methods and substances to improve lung graft preservation during cold ischemia, such as pulmonary surfactant, liquid perfluorocarbon (PFC), complement inhibitors, and nitrites, as well as inhalation of hydrogen sulfide or nitric oxide, have been tested experimentally. ${ }^{(1,2)}$ However, the standard method of lung graft preservation is still perfusion with a cold low-potassium dextran (LPD) solution via the pulmonary artery (antegrade perfusion) or via the pulmonary veins (retrograde perfusion), together with the use of pulmonary vasodilators and partial inflation of the lungs with oxygen during storage. ${ }^{(2)}$ The use of vaporized PFC seems to be an interesting alternative in lung graft preservation, given that the use of liquid PFC has been shown to be protective of lung grafts before and after reperfusion. ${ }^{(3-5)}$ The potential benefits of vaporized PFC in lung graft preservation are its ability to transport oxygen and carbon dioxide, together with its anti-inflammatory and antioxidant properties. ${ }^{(6-8)}$ In addition, in its vaporized form, PFC is easily distributed throughout the lungs in a more uniform way, and, chiefly, it does not cause the ventilation difficulties observed in lungs perfused with liquid PFC. To determine the effects of vaporized PFC during lung graft preservation and to analyze oxidative stress/histological changes in lung grafts preserved for different periods of time, we used an animal model of cold ischemia. This was a controlled experimental study involving Wistar rats with a mean body weight of $300 \mathrm{~g}$. All animals were treated in accordance with the World Health Organization Code of Ethics for Animal Experimentation. The animals were divided into two groups, each of which was subdivided into four groups. Each subgroup comprised six animals, depending on the surgical procedure: PFC + LPD 3h; PFC + LPD 6h; PFC + LPD 12h; and PFC + LPD 24h subgroups vs. LPD 3h; LPD 6h; LPD 12h; and LPD 24h. In the four PFC + LPD subgroups, regardless of preservation time, we used a dose of $7 \mathrm{~mL} / \mathrm{kg}$ of vaporized PFC, through a tracheotomy cannula connected to an anesthesia machine, after a $120-$ min period of reperfusion. The animals were killed after anesthesia with ketamine (100 $\mathrm{mg} / \mathrm{kg}$, i.p.) and xylazine (50 mg/kg). Subsequently, a mid-ventral laparotomy was performed. The lungs were removed and fixed in 4\% paraformaldehyde for histological analysis and were stored at $-80^{\circ} \mathrm{C}$ for subsequent quantification of thiobarbituric acid reactive substances (TBARS), as well as for evaluation of the activity of the enzymes superoxide dismutase (SOD) and catalase. For biochemical analysis, the lung tissue was homogenized, after which protein levels were quantified as proposed by Lowry et al.(9) The levels of TBARS were measured as described by Buege and Aust, ${ }^{(10)}$ and SOD activity was determined according to

Correspondence to:

Renata Salatti Ferrari. Rua Ramiro Barcelos, 2350, Santa Cecília, CEP 90035-903, Porto Alegre, RS, Brasil.

Tel.: 5551 99636-6999. E-mail: renatasalattiferrari@gmail.com

Financial support: This study received financial support from the Fundo de Incentivo à Pesquisa (FIPE, Research Incentive Fund) of the Porto Alegre Hospital de Clínicas (Protocol no. 120430) 
the technique described by Misra and Fridovich.(11) Catalase activity was analyzed by measuring the reduction in hydrogen peroxide levels. ${ }^{(12)}$ The lung tissue samples for histological analysis were collected and stored in $10 \%$ formaldehyde solution for $12 \mathrm{~h}$, after which the solution was replaced with $70 \%$ alcohol and the samples were stained with H\&E. All pathology studies were performed by a pathologist who was blinded to the subgroups. Data were analyzed with IBM SPSS Statistics software, version 22.0 (IBM Corporation, Armonk, NY, USA). ANOVA followed by Tukey's post hoc test was used; in cases of unequal variance or non-normal distribution, the nonparametric Kruskal-Wallis test was performed, followed by the Mann-Whitney $U$ test for intergroup comparisons. For all comparisons, the level of significance was set at $5 \%$. The lungs preserved with vaporized PFC (at a dose of $7 \mathrm{~mL}$ / $\mathrm{kg})+$ LPD for 3 and $6 \mathrm{~h}$ showed significantly higher SOD concentrations than did those in the LPD $3 \mathrm{~h}$ and LPD 6h subgroups, respectively. We found no significant differences in TBARS or catalase levels among the subgroups (Figure 1).

On histology, we observed the presence of interstitial infiltrate, chronic interstitial inflammation, and atelectasis in the lungs in the LPD $3 \mathrm{~h}$, LPD $6 \mathrm{~h}$, LPD $12 \mathrm{~h}$, and LPD $24 \mathrm{~h}$ subgroups, as determined by intravascular macrophage counts. In the corresponding PFC + LPD subgroups, we observed only the presence of atelectasis, which demonstrates that the use of vaporized PFC reduced pulmonary structural damage at different cold ischemia times (Figure 2).
Our results show that the use of vaporized PFC concomitantly with mechanical ventilation reduced oxidative stress during an initial cold ischemia period (for lung graft preservation) of up to $6 \mathrm{~h}$, thus proving its antioxidant effect. Similar findings were obtained by Forgiarini Junior et al.,(4) who evaluated the effects of liquid PFC in a rat model of lung transplantation. In that study, the authors evaluated oxidative stress at different ischemia times and after lung transplantation, finding an increase in SOD activity but no significant differences in TBARS levels. ${ }^{(4)}$ Liquid PFC has the characteristic of maintaining the alveolar structure, even after lung injury in a model of ischemia/reperfusion injury by clamping the pulmonary hilum ${ }^{(3)}$ or in a model of lung transplantation. (4) Forgiarini Junior et al. ${ }^{(4)}$ tested different doses of liquid PFC and demonstrated that, by using a dose of $7 \mathrm{~mL} / \mathrm{kg}$, there was better maintenance of the alveolar structure without rupture of alveolar septa. Our study demonstrated that, even in the vapor state, PFC has properties similar to those of liquid PFC in terms of protection of the alveolar structure. Although the results of our study are preliminary, to our knowledge, this is the first time that vaporized PFC has been tested as an adjuvant therapy in the preservation of lung grafts during cold ischemia. Our findings suggest that it provides protection to the alveolar structure and has antioxidant properties. Further studies are required in order to define the actual role of vaporized PFC in lung graft preservation and in the reperfusion phase after transplantation.

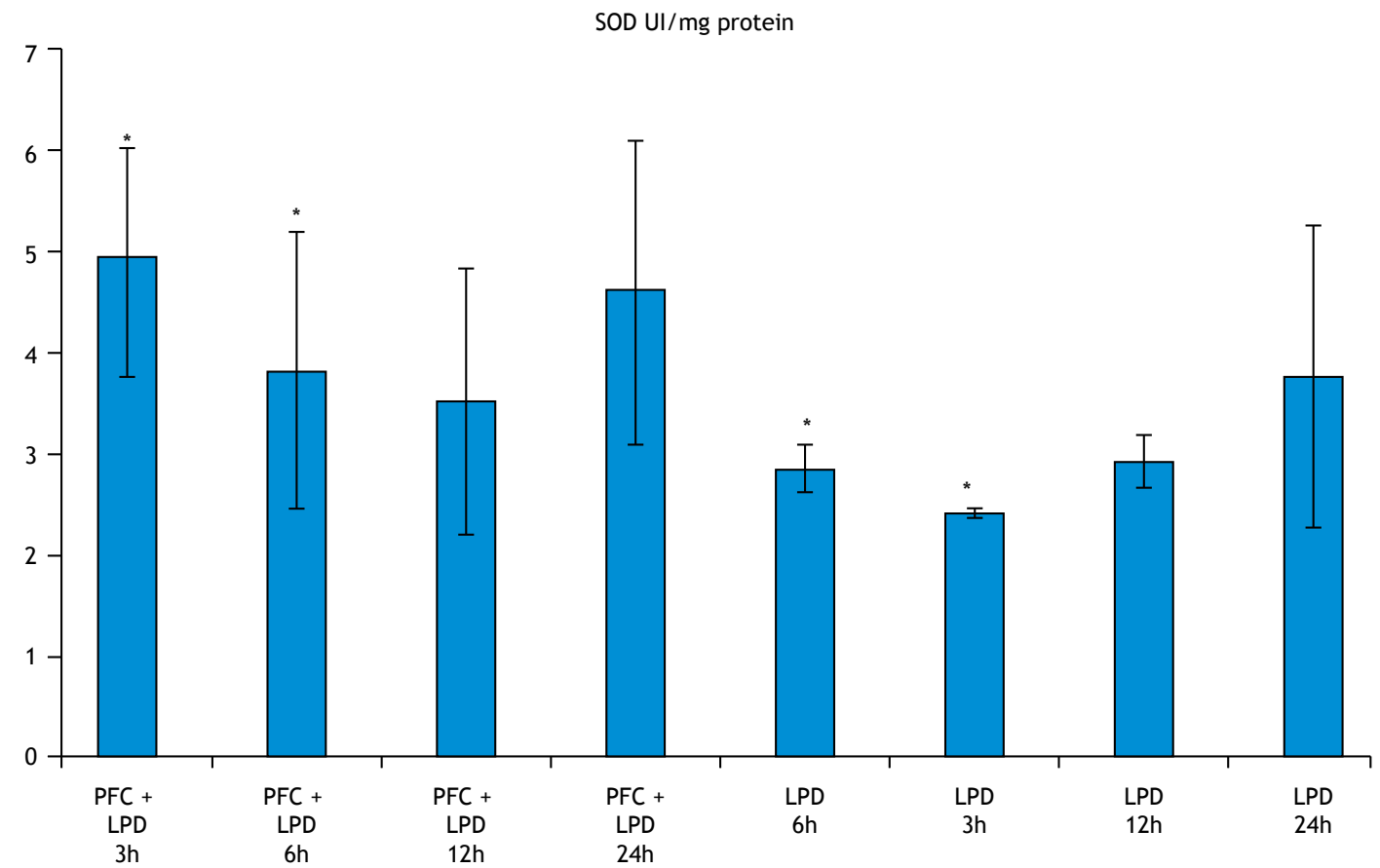

Figure 1. Comparison between the perfluorocarbon (PFC) + low-potassium dextran (LPD) subgroups and the LPD-only subgroups regarding the activity of the enzyme superoxide dismutase (SOD). Values expressed as mean $\pm \mathrm{SD}$. ${ }^{*} \mathrm{p}<$ 0.05 (PFC + LPD 3h vs. LPD 3h; and PFC + LPD 6h vs. LPD 6h). 


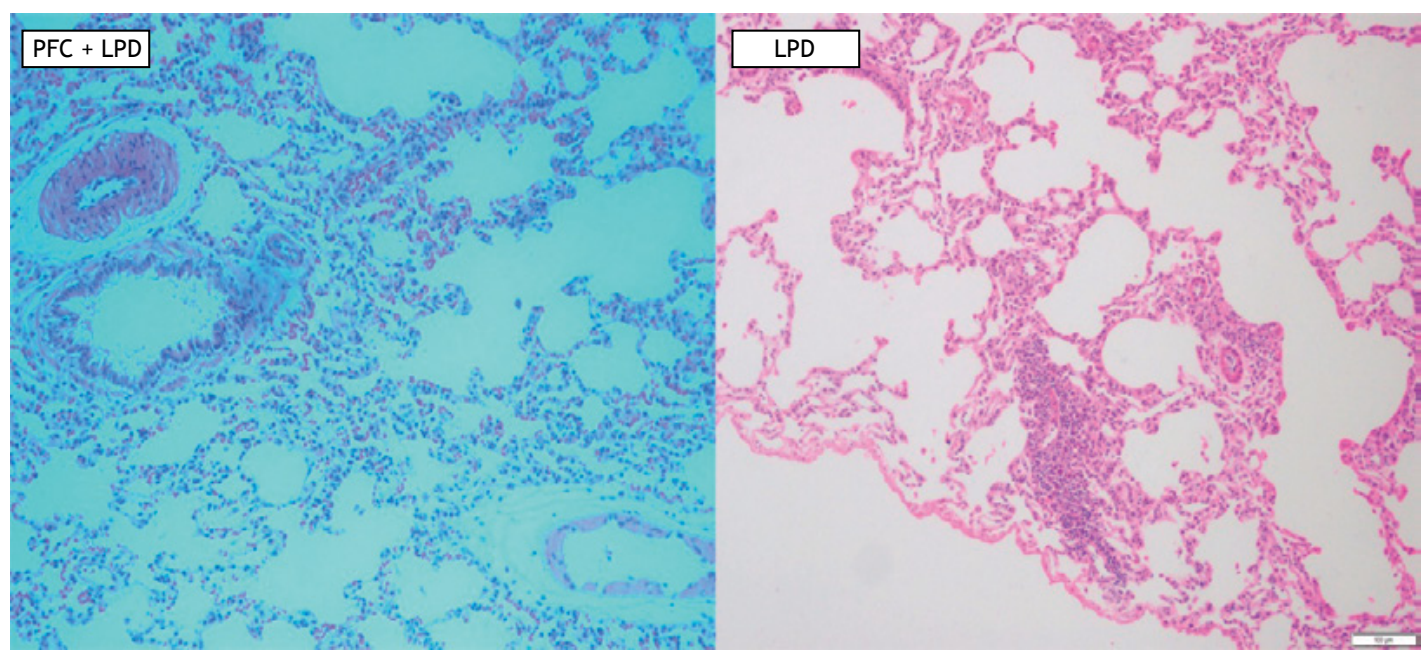

Figure 2. Photomicrographs of lung tissue samples after $24 \mathrm{~h}$ of cold ischemia. There is greater inflammation in the lungs that were perfused with low-potassium dextran (LPD) than in the lungs treated with vaporized perfluorocarbon $(P F C)+$ LPD. (H\&E; magnification, $\times 100)$

\section{REFERENCES}

1. Cypel M. A new era in lung transplantation: an individualized approach to donor lungs. J Bras Pneumol. 2012;38(6):681-3. https:// doi.org/10.1590/S1806-37132012000600001

2. Andrade CF, Kaneda H, Der S, Tsang M, Lodyga M, Chimisso Dos Santos C, et al. Toll-like receptor and cytokine gene expression in the early phase of human lung transplantation. J Heart Lung Transplant. 2006;25(11):1317-23. https://doi.org/10.1016/j.healun.2006.09.017

3. Forgiarini LA Jr, Forgiarini LF, da Rosa DP, Mariano R, Ulbrich JM, Andrade CF. Endobronchial perfluorocarbon administration decreases lung injury in an experimental model of ischemia and reperfusion. J Surg Res. 2013;183(2):835-40. https://doi. org/10.1016/j.jss.2013.01.035

4. Forgiarini Junior LA, Holand AR, Forgiarini LF, da Rosa DP, Marroni $N A$, Cardoso PF, et al. Endobronchial perfluorocarbon reduces inflammatory activity before and after lung transplantation in an animal experimental model. Mediators Inflamm. 2013;2013:193484. https://doi.org/10.1155/2013/193484

5. Ferrari RS, Andrade CF. Oxidative Stress and Lung IschemiaReperfusion Injury. Oxid Med Cell Longev. 2015;2015:590987.

6. Wang X, Zhang J, Li X, Liu Y, Yang H, Zhao X, et al. Sustained improvement of gas exchange and lung mechanics by vaporized perfluorocarbon inhalation in piglet acute lung injury model. Clin
Respir J. 2014;8(2):160-6. https://doi.org/10.1111/crj.12053

7. Hübler M, Souders JE, Shade ED, Polissar NL, Schimmel C, Hlastala MP. Effects of vaporized perfluorocarbon on pulmonary blood flow and ventilation/perfusion distribution in a model of acute respiratory distress syndrome. Anesthesiology. 2001;95(6):1414-21. https://doi. org/10.1097/00000542-200112000-00021

8. de Abreu MG, Quelhas AD, Spieth P, Bräuer G, Knels L, Kasper M et al. Comparative effects of vaporized perfluorohexane and partial liquid ventilation in oleic acid-induced lung injury. Anesthesiology. 2006;104(2):278-89. https://doi.org/10.1097/00000542-20060200000013

9. LOWRY OH, ROSEBROUGH NJ, FARR AL, RANDALL RJ. Protein measurement with the Folin phenol reagent. J Biol Chem 1951;193(1):265-75.

10. Buege JA, Aust SD. Microsomal lipid peroxidation. Methods Enzymol. 1978;52:302-10. https://doi.org/10.1016/S0076-6879(78)52032-6

11. Misra HP, Fridovich I. The role of superoxide anion in the autoxidation of epinephrine and a simple assay for superoxide dismutase. J Bio Chem. 1972;247(10):3170-5.

12. Aebi H. Catalase in vitro. Methods Enzymol. 1984;105:121-6. https:// doi.org/10.1016/S0076-6879(84)05016-3 